\title{
Unsupervised CNN model for Sclerosis Detection
}

\author{
Ms. K. N. Rodea, and Dr. Rajshekhar J S ${ }^{b}$ \\ a \\ Research Scholar, VTU University, DSCE, Bangalore, Assist Profes-sor Sharad \\ Institute of Technology, College of Engineering. \\ bProfessor \& Head, Dept. of IT, DSCE, Bangalore.
}

Article History: Received: 11 January 2021; Accepted: 27 February 2021; Published online: 5 April 2021

\begin{abstract}
Sclerosis detection using brain magnetic resonant imaging (MRI) im-ages is challenging task. With the promising results for variety of ap-plications in terms of classification accuracy using of deep neural net-work models, one can use such models for sclerosis detection. The fea-tures associated with sclerosis is important factor which is highlighted with contrast lesion in brain MRI images. The sclerosis classification initially can be considered as binary task in which the sclerosis segmentation can be avoided for reduced complexity of the model. The sclerosis lesion show considerable impact on the features extracted us-ing convolution process in convolution neural network models. The images are used to train the convolutional neural network composed of 35 layers for the classification of sclerosis and normal images of brain MRI. The 35 layers are composed of combination of convolutional lay-ers, Maxpooling layers and Upscaling layers. The results are com-pared with VGG16 model and results are found satisfactory and about $92 \%$ accuracy is seen for validation set.
\end{abstract}

Keywords: CNN, brain MRI, vgg16, unsupervised, deep learning, features.

\section{Introduction}

Presence of sclerosis is detected with the help of locating lesions in brain MRI image. The lesions present in the MRI image are the main factor considered to identify the sclerosis. The semantic segmentation of lessions is the complex task and in case of simply scelerosis detec-tion objective segmentation can be avoided and complexity of pro-cessing can be reduced. Many classical methods have shown the spa-tial and textural features extraction using hand crafted rules for the identification of scelerosis but classification accuracy ultimately depends on neural network classifier convergence capabilityies. Here the point to be noted that, features obtained through hand crafted rule are the main cause that define the convergence capability of the neural network and hence limitation in accuracy levels achievements are seen. On the other hand deep neural networks can be used for both feature extraction and classification purposes and the accuracy of classification can be improved. The convolutional neural network based models such as VGG16, VGG19 are available for the classifica-tion task for the images having single objects and less crwody infor-mation contents. But these models fails to classify the scelerosis im-ages correctly and the ultimate goal of accuracy remains unsatsfaied. When these models are compared with ResNets having 50 or 100 lay-ers in the model then intuitively number of layers in VGG16 can be increased for accuracy improvement. But as the number of layers goes on increasing along with them Maxpooling layers also increase and fi-nal image size during the processing becomes so small further that convergence achievement fails and hence shows the poort perfor-mance. Hence simply increasing the layers in base VGG16 model shows less improvements and lossy outcomes in terms of validation accuracies.

So far, classical approaches have also focused on object seg-mentation and then listing for recognizing sclerosis images. Over these classic methods, convolutional neural networks are also required to be trained specifically with respect to objects in the images.

This work contributes in development of 35 layered convolu-tional neural network (CNN) model for classification of sclerosis im-ages in unsupervised manner. The unsupervised word here is specifi-cally applicable for avoidance of segmentation and not using the ground truth information about coordinates of lesion regions in the sclerosis MRI images.

\section{Related Work}

Among every neurological illness, numerous sclerosis (con-tracted as MS) is a condition influencing mind or potentially spinal rope [1]. It might prompt a scope of possible manifestations, for ex-ample, development [2], vision[3], balance[4], and so on Customary conclusion strategies dependent on introducing manifestations. These days, imaging strategy, e.g., magnetic resonant imaging (MRI) proce-dures are ordinarily used to distinguish the foci in white matter of the MS patients, giving a quantitative finding technique. A few treat-ments like interferon $\beta-1 \mathrm{a}$, natalizumab, and alemtuzumab that stifle or balance assorted safe framework capacities have been utilized for quite a long time with the point of altering the illness course. In any case, these medicines have either restricted viability or conceivably genuine unfriendly occasions that forestall first-line use for huge scope. MS 
determination might be mistaken for other white issue ail-ments, for example, intense cerebral dead tissue[5], intense dispersed encephalomyelitis (ADEM), neuromyelitisoptica (NMO)[6], etc.

In this paper, techniques are discussed that recognizes MS from solid controls with the assistance of MRI. As machines normally acquire preferable outcomes over natural eyes, researchers will in gen-eral utilize PC vision [7, 8] procedures to understand this undertaking. For occasions, Murray, Rodriguez (2010) [9] presented a multiscale adequacy tweak recurrence adjustment (MAMSM) approach. Zhou (2016) [10] used fixed wavelet entropy (SWE) as highlight descriptor. They looked at three classifiers: choice tree, k-closest neighbors (kNN), and backing vector machine. They discovered kNN acquired the most elevated exactness. Zhan and Chen (2016) [11] utilized part head segment investigation (KPCA). Lopez (2017) [12] consolidated Haarwavelet change (HWT) with calculated relapse (LR). Their analy-sis approved three-level wavelet disintegration accomplished the ideal presentation. Ghribi, Sellami (2018) [13]presentedhybrid technique consolidated bothgray-level coevent grid (GLCM) and dark level run length (GLRL) strategies.

Cheng (2018) [14] proposed a changed Jaya calculation to dis-tinguish MS patients.Atangana (2018) [15] recommended to utilize fixed wavelet entropy (SWE).

Chen (2017) [16] utilized direct relapse classifier (LRC). Chen and Chen (2016) [17] utilized Tikhonov regularization approach. Those cutting edge approaches have gotten promising outcomes. By and by, they are powerless against following issues. Firstly datasets were imbalanced, since MS information are more hard to gather. Sec-ond, the element descriptors in their techniques were usually acquired by manual test. Third, their classifiers were straightforward, and may not extract the convoluted MS model. Our commitment in this investigation for the most part centers around utilizing profound figuring out how to recognize MS from sound control (HC). The convolutional neural organization $(\mathrm{CNN})$ is a hotspot device inside the field of pro-found learning, and it has been effectively applied to numerous scho-lastic fields, e.g., far off detecting division [18], contract default iden-tification [19], pearl grouping [20], cerebral microbleeding [21], and so on For the most part, CNN can be utilized in design acknowledg-ment, recognition, assessment [27], expectation [28], relapse, and so on In their papers, $\mathrm{CNN}$ was accounted for to increase preferred exe-cution over common PC vision draws near.

Our technique can help distinguish MS from solid individuals. This can help neuroradiologists to have a coarse finding on a given at-tractive reverberation mind picture. At that point, the neuroradiolo-gists can get an opportunity to second-check the cerebrum picture, and assess the seriousness of the MS infection. Later on research, this subsequent check is relied upon to be actualized consequently by means of CNN either.

\section{Proposed Work}

The work is proposed by considering following assumptions.

1. The model should classify the images in sclerosis and normal images.

2. There should be avoidance of segmentation requirement and totally unsupervised approach should be used.

3. Maximum accuracy in classification of images should be the main objective.

The proposed work consists of two phases of work. The first phase consist of dataset preprocessing and second phase focuses on model development, implementation and classification.

Figure 1 shows the block diagram of the proposed work. Each input image is firstly preprocessed and then passed through con-volution neural network layers for feature extraction and classifi-cation.

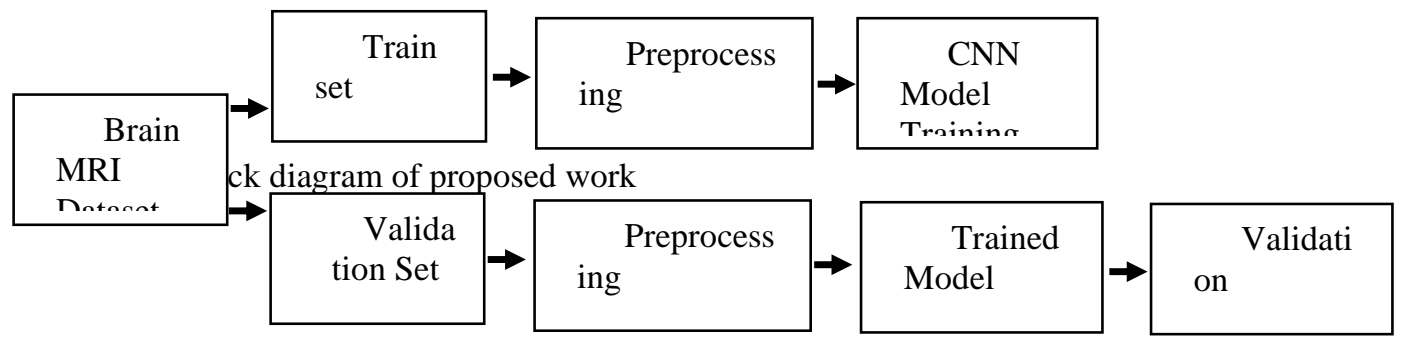

Figure 2 shows the example of brain MRI image with lesion in or-gan sliced from multiple Sclerosis dataset [22]. 


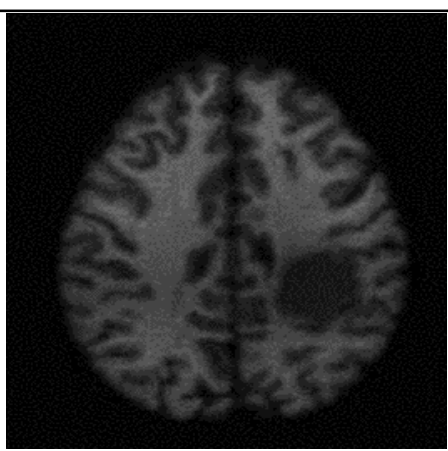

Model development

Figure 1: Sliced image from multiple Sclerosis dataset

The proposed work consists of developing CNN model archi-tecture for sclerosis image classification. The model architecture is shown in figure 2 .

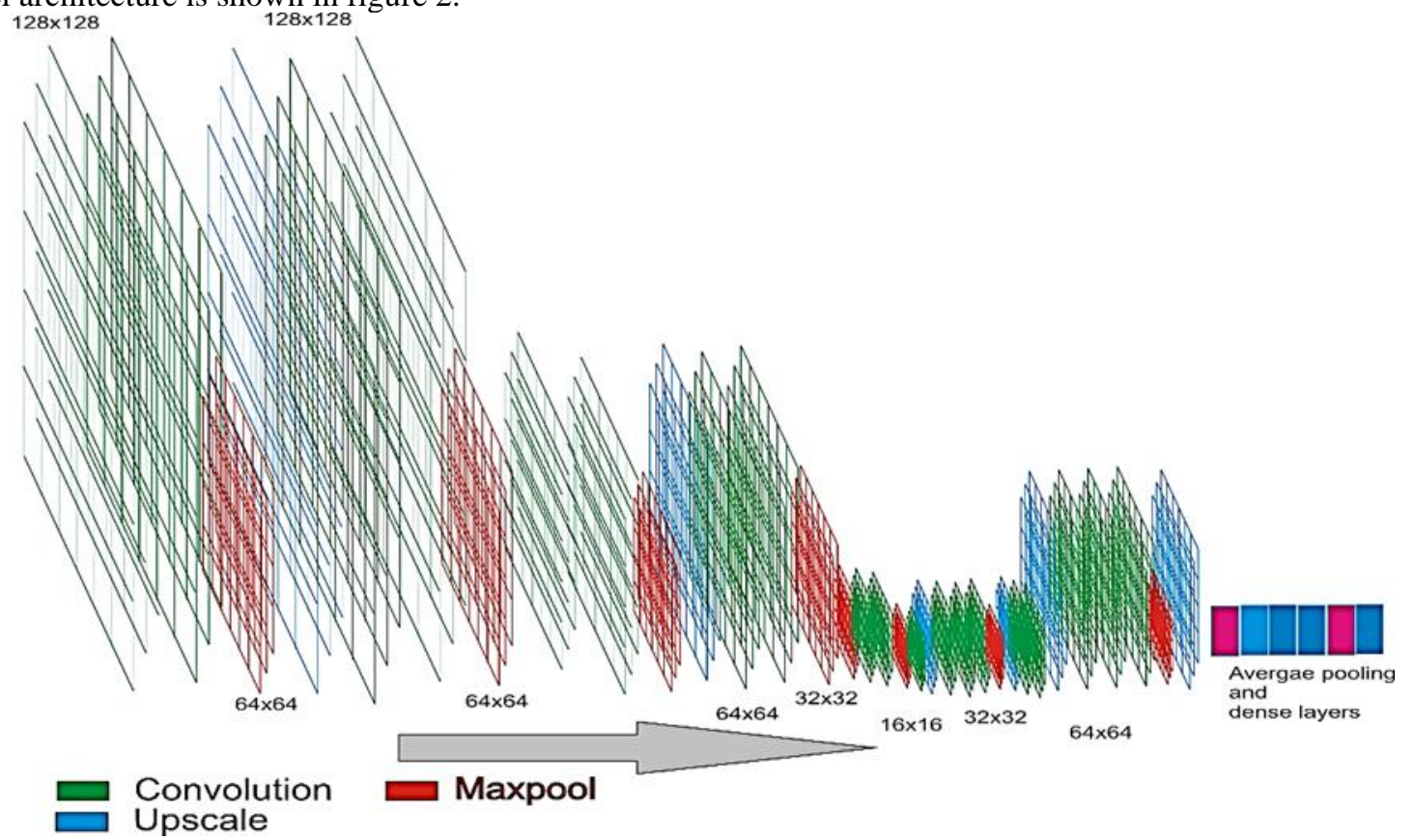

Figure 2: Proposed CNN model architecture

The proposed 35 layered model is shown in figure 2 . When image input size is up to $128 \times 128$ or less, increment in layers leads to loss of scene details and continues on focusing single object characteristics. This leads to the model type which is similar to VGG16 and increasing layer even in VGG16 for modification shows similar lossy progress. To avoid this loss and keep scenery characteristics paired together while retrieving features of the im-age, it becomes necessary to expand the image using up scaling. Also, up scaling as per routine principle increases the details of the features. In most of the medical image segmentation algorithms such as UNet, up scaling plays important role for detailed feature analysis during segmentation. This principle can be considered to enhance the scene details without concatenation and further inclu-sion of downscaling operations.

In many semantic segmentation-based methods using CNN models, there is use of Up scaling layers to maximize the details of the objects in the image while performing scene recognition. Based on the assumptions highlighted in this work, the segmenta-tion requirement is totally avoided but at the same time to increase the details of the features with respect to the objects, there is in-clusion of up scaling layers in our model which improved the per-formance and avoids lossy structure of the feature sets. Also, it does not require any concatenation or merging of the feature maps as in semantic segmentations.

The proposed model performance is compared with VGG16 mod-el. The VGG16 model is modified by changing classes in last dense layer by setting its value to 2 . The model is trained on the same dataset that is used for proposed model training.

\section{Results and analysis}

The proposed model is implemented using TensorFlow, Keras tools using python programming. The training is performed with 100 epochs and 10 steps per epoch. The machine configuration used for experimentation is consist of Intel i7 10th gen processor, 32GB DDR4 RAM and 6GB Nvidiagtx 1660ti graphics proces-sor.

The feature maps are obtained by training the proposed architec-ture. The outcomes of layer number 20,30 and 32 in terms of $4 \times 4$ feature maps are shown in figure 3. 


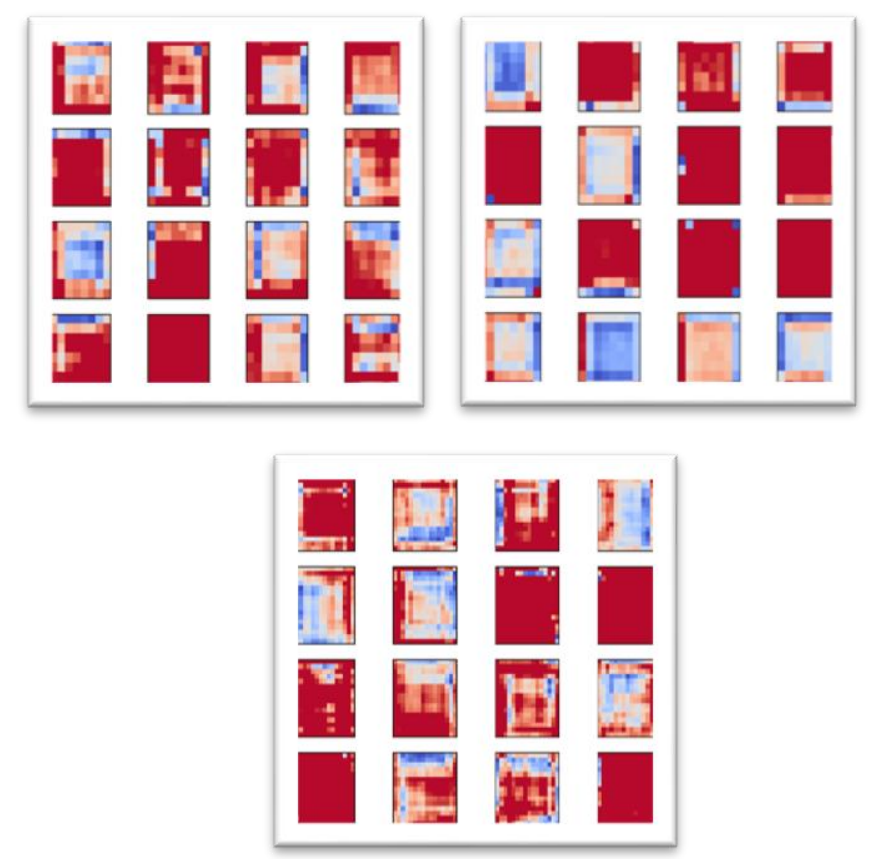

Figure 3: Feature maps obtained at layers 20,30 and 32 in terms of $4 \times 4$ maps

Loss rate analysis:

The loss analysis is shown in Figure 4 for total 1000 steps.

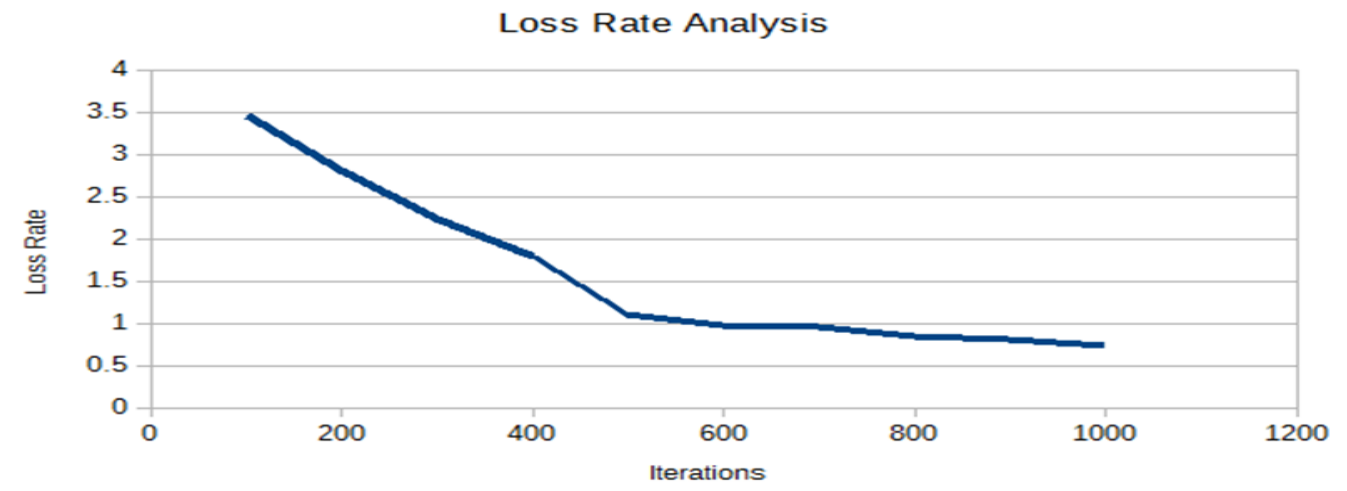

Figure 4: Loss rate analysis

Accuracy analysis

The accuracy analysis is performed for different number of epochs in which 100 epochs show better performance and as minimum level of required for the training. Table 1 and figure 5 show accu-racy analysis. The retrained VGG16 model performance is also evaluated in similar manner.

Table 1: Accuracy analysis

\begin{tabular}{|c|c|c|}
\hline $\begin{array}{c}\text { Number of epochs in } \\
\text { training }\end{array}$ & $\begin{array}{c}\text { Proposed } \\
\text { Model Accuracy } \\
(\%)\end{array}$ & $\begin{array}{c}\text { VGG16 } \\
\text { Accuracy(\%) }\end{array}$ \\
\hline 30 & 79.23 & 76.44 \\
\hline 50 & 82.45 & 80.10 \\
\hline 70 & 85.1 & 84.66 \\
\hline 100 & 89.33 & 87.91 \\
\hline
\end{tabular}




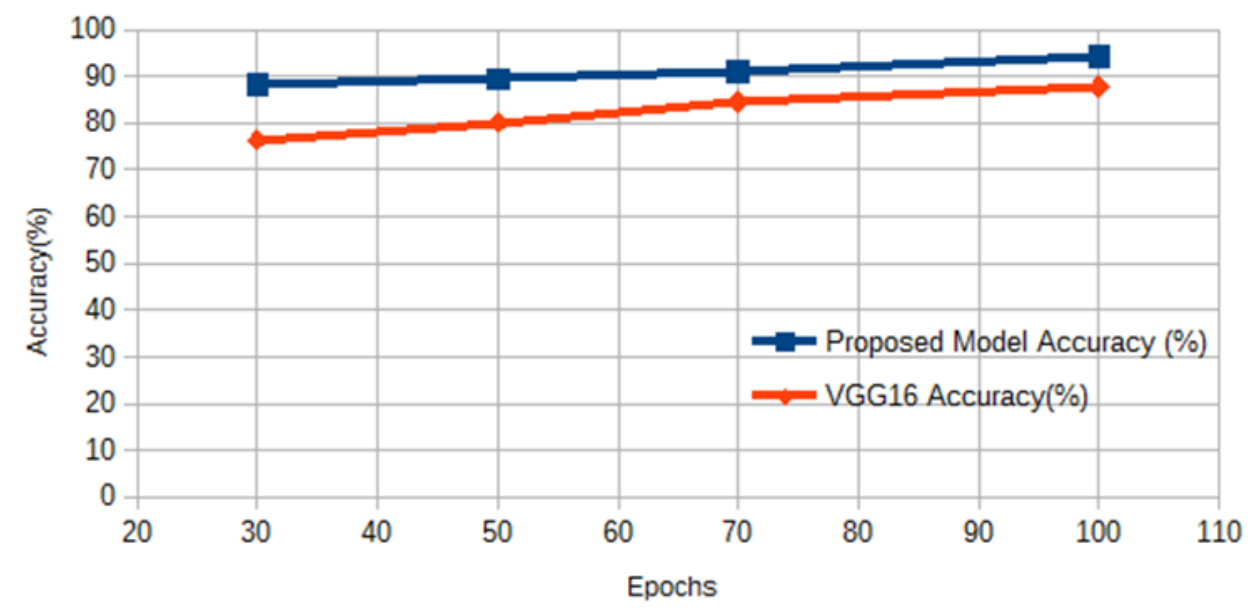

\section{Conclusion}

Figure 5: Accuracy analysis with respect to training epochs

This positional paper contributes in terms of unsupervised CNN model for binary classification of large image dataset in terms of sclerosis and normal classes. This model does not require scene segmentation in semantic manner and hence reduced complexity of architecture. The performance of model is up to $89 \%$ which better than VGG16 model. Also, maximum details preservation is the main processing requirement while performing max pooling and convolutional operations is the main concluding remark while increasing the number of layers in the deep network model. The results obtained on single dataset experimentation are satisfactory and also shows further path for evaluating more combinations of different datasets with different constraints as matter of preprocessing requirements and further modifications in the model archi-tecture which may constitute use of attention models

\section{References}

1. Dolati, S., et al., Nanocurcumin restores aberrant miRNA ex-pression profile in multiple sclerosis, randomized, double blind, placebocontrolled trial. Journal of Cellular Physiology, 2018. 233 (7): p. 52225230

2. Hatteb, S.A., et al., Movement disorders in a cohort of Algeri-an patients with multiple sclerosis. Revue Neurologique, 2018. 174 (3): p. 167-172

3. Sanchez Dalmau, B., et al., Predictors of vision impairment in Multiple Sclerosis. Plos One, 2018. 13 (4): p. 12: Article ID. e0195856

4. Tona, F., et al., Role of Cerebellar Dentate Functional Connec-tivity in Balance Deficits in Patients with Multiple Sclerosis. Radiology, 2018.287 (1): p. 267275

5. Deguchi, I., et al., Clinical Study of Intravenous, Low Dose Recombinant Tissue Plasminogen Activator for Acute C ere-bral Infarction:Comparison of Treatment within 3 Hours ver-sus 34.5 Hours. Journal of Stroke \& Cerebrovascular Diseas-es, 2018. 27 (4): p. 10331040

6. Lana Peixoto, M.A., et al., Neuromyelitisoptica spectrum dis-order associated with dengue virus infect ion. Journal of Neu-roimmunology,2018. 318: p. 53-55

7. Pan, H., et al., RGB D image based detection of stairs, pedes-trian crosswalks and traffic signs. Journal of Visual Communi-cation and ImageRepresentation, 2014. 25 (2): p. 263-272

8. Liu, A.J., Tea Cat egory Identification using Computer Vision and Generalized Eigenvalue Proximal SVM. FundamentaIn-formaticae, 2017.

9. Murray, V., et al., Murray, V., et al., Multiscale AMMultiscale AM--FM Demodulation and Image Reconstruction Methods With Improved Accuracy.FM Demodulation and Image Re-construction Methods With Improved Accuracy. IEEE Tran-sIEEE Transactions on actions on Image Processing, 2010. Image Processing, 2010. 1919(5): p. 1138(5): p. 1138--11521152

10. Zhou, X.Zhou, X.--X., X., Comparison of machine learning methods for stationary wavelet entropyComparison of ma-chine learning methods for stationary wavelet entropy--based multiple sclerosis detection: decision tree, kbased multiple sclerosis detection: decision tree, $k$--nearest neighbors, and support vector machine.nearest neighbors, and support vector machine. Simulation, 20Simulation, 2016. 16. 9292(9): p. 861(9): p. 861--871871

11. Zhan, T.M., et al., Zhan, T.M., et al., Multiple Sclerosis De-tection Based on Biorthogonal Wavelet Transform, RBF Ker-nel Principal Component Analysis, and Multiple Sclerosis De-tection Based on 
Biorthogonal Wavelet Transform, RBF Ker-nel Principal Component Analysis, and Logistic Regression.Logistic Regression. IEEE Access, 2016. IEEE Access, 2016. 44: p. 7567: p. 7567--75767576

12. Lopez, M., Lopez, M., Multiple Sclerosis SliceMultiple Scle-rosis Slice Identification by Haar Wavelet Transform and Lo-gistic Regression.Identification by Haar Wavelet Transform and Logistic Regression. Advances in Engineering Research, Advances in Engineering Research, 2017. 2017. 114114: p. 50: p. 50--5555

13. Ghribi, O., et al., Ghribi, O., et al., Multiple sclerosis explora-tion based on automatic MRI modalities segmentation ap-proach with advanced Multiple sclerosis exploration based on automatic MRI modalities segmentation approach with ad-vanced volumetric volumetric evaluations for essential feature extraction.evaluations for essential feature extraction. Biomed-ical Signal Processing and Control, 2018. Biomedical Signal Processing and Control, 2018. 4040: p. 473: p. 473--487487

14. Cheng, H., Cheng, H., Multiple sclerosis identification based on fractional Fourier entropy and a modified Jaya algo-rithm.Multiple sclerosis identification based on fractional Fou-rier entropy and a modified Jaya algorithm. Entropy, 2018. Entropy, 2018. 2020(4)(4): Article : Article ID. 254ID. 254

15. Atangana, A., Atangana, A., Application of stationary wave-let entropy in pathological brain detection.Application of sta-tionary wavelet entropy in pathological brain detection. Mul-timedia Tools and Applications, 2018. Multimedia Tools and Applications, 2018. 7777(3): p. (3): p. 37013701 -37143714

16. Chen, Y., Chen, Y., A FeatureA Feature--Free 30Free 30--Disease Pathological Brain Detection System by LineaDisease Pathological Brain Detection System by Linear Regression Classifier.r Regression Classifier. CNS \& Neurological Disor-ders CNS \& Neurological Disorders -- Drug Targets, 2017. Drug Targets, 2017. 1616(1): p. 5(1): p. 5--1010

17. Chen, Y., et al., Chen, Y., et al., Sensorineural hearing loss detection via discrete wavelet transform and principal compo-nent analysis combined with Sensorineural hearing loss detec-tion via discrete wavelet transform and principal component analysis combined with generalized eigenvalue proxigeneral-ized eigenvalue proximal support vector machine and Tikhonovregularization.mal support vector machine and Tikhonov regularization. Multimedia Tools and Applications, 2016. Multimedia Tools and Applications, 2016. 7777(3): p. (3): p. 37753775--37933793

18. Zhao, G.Zhao, G.,, Polarimetric synthetic aperture radar im-age segmentation by convolutional neural network using graphical processing unitsPolarimetric synthetic aperture radar image segmentation by convolutional neural network using graphical processing units. . JoJournal of Realurnal of Real--Time Image Processing, 2017, doi: 10.1007/s11554Time Im-age Processing, 2017, doi: 10.1007/s11554-. 017017--07170717--0.0.

19. Kvamme, H., et al., Kvamme, H., et al., Predicting mortgage default using convolutional neural networks.Predicting mort-gage default using convolutional neural networks. Expert Sys-tems with Applications, 2018. Expert Systems with Applica-tions, 2018. 102102: p. 207: p. 207--217217

20. Xuan, Q., et al., Xuan, Q., et al., Automatic PearAutomatic Pearl Classification Machine Based on a Multistream Convolu-tional Neural Network.l Classification Machine Based on a Multistream Convolutional Neural Network. IEEE Transac-tions on IEEE Transactions on Industrial Electronics, 2018. Industrial Electronics, 2018. 6565(8): p. 6538(8): p. 6538--65476547

21. Jiang, Y.Y., Jiang, Y.Y., Cerebral MicroCerebral Micro--Bleed Detection Based on the Convolution Neural Network With Rank Bleed Detection Based on the Convolution Neural Network With Rank Based Average Pooling.Based Average Pooling. IEEE Access, IEEE Access, 2017. 2017. 55: p. 16576: p. 16576--1658316583

22. MRI Lesion Segmentation in Multiple Sclerosis Database, in eHealth laboratory, University of Cyprus, Available from: MRI Lesion Segmentation in Multiple Sclerosis Database, in eHealth laboratory, University of Cyprus, Available from: http://www.medinfo.cs.ucy.ac.cy/index.php/downloads/datasetshttp://www.medinfo.cs.ucy.ac.cy/index.p hp/downloads/datasets.

23. Kim, D., Contrast Enhancement Scheme using Histogram Stretching and Equalization on Singular Value Domain. In-formation, 2017. 20 (2B):p. 1221-24. 
24. Lee, L.C., et al., Validity of the best practice in splitting data for hold out validation strategy as performed on the ink strokes in the context of forensic science. Microchemical Jour-nal, 2018. 139: p. 125133.

25. Touloupou, P., et al., Efficient Model Comparison Tech-niques for Models Requiring Large Scale Data Augmentation. Bayesian Analysis, 2018. 13 (2): p. 437459 20-26.

26. Lv, Y.Lv, Y.--D., D., Alcoholism detection by data augmen-tation and convolutional neural network with stochastic pool-ing.Alcoholism detection by data augmentation and convolu-tional neural network with stochastic pooling. Journal of Med-ical Systems, Journal of Medical Systems, 2018. 2018. 4242(1): Article ID. 2(1): Article ID. 2-27.

27. Li, W.GLi, W.G., ., First and Others creditFirst and Others credit--assignment schema for evaluating the academic contri-bution of coauthors.assignment schema for evaluating the aca-demic contribution of coauthors. Frontiers of Information Frontiers of Information Technology \& Electronic Engineer-ing, 2017. Technology \& Electronic Engineering, 2017. 1818(2): p. 180(2): p. 180-194194-28.

28. Li, W.G. Li, W.G. Flexible Schema for Prediction of Collab-orator's CreditsFlexible Schema for Prediction of Collabora-tor's Credits.. in in 9th International Conference on Security, Privacy, and Anonymity in 9th International Conference on Security, Privacy, and Anonymity in Computation, Communi-cation and Storage (SpaCCS)Computation, Communication and Storage (SpaCCS). 2016. Zhangjiajie, China: Springer Int Publishing Ag. p. 218. 2016. Zhangjiajie, China: Springer Int Publishing Ag. p. 218-228228

29. Dr.A.Senthil Kumar, Dr.G.Suresh, Dr.S.Lekashri, Mr.L.Ganesh Babu, Dr. R.Manikandan. (2021). Smart Agri-culture System With E - Carbage Using Iot. International Journal of Modern Agriculture, 10(01), 928 - 931. Retrieved from http://www.modern-journals.com/index.php/ijma/article/view/690

30. Dr.G.Suresh, Dr.A.Senthil Kumar, Dr.S.Lekashri, Dr.R.Manikandan. (2021). Efficient Crop Yield Recommen-dation System Using Machine Learning For Digital Farming. International Journal of Modern Agriculture, 10(01), 906 - 914. Retrieved from http://www.modernjournals.com/index.php/ijma/article/view/688

31. Dr. R. Manikandan, Dr Senthilkumar A. Dr Lekashri S. Ab-hay Chaturvedi. "Data Traffic Trust Model for Clustered Wireless Sensor Network. ” INFORMATION TECHNOLOGY IN INDUSTRY 9.1 (2021): 1225-1229. Print. 